\title{
A mathematical model for the diagnosis and treatment of idiopathic intracranial hypertension
}

\author{
W. D. Lakin ${ }^{1}$, S. A. Stevens ${ }^{2}$, N. J. Thakore ${ }^{3}$ \& P. L. Penar ${ }^{1}$ \\ ${ }^{1}$ The University of Vermont, USA \\ ${ }^{2}$ School of Science, Penn State Erie, The Behrend College, USA \\ ${ }^{3}$ MetroHealth Medical Center and Case Western Reserve University, USA
}

\begin{abstract}
Idiopathic intracranial hypertension (IIH) is a syndrome of unknown cause characterized by elevated intracranial pressure (ICP). A stenosis of the venous sinus is observed in many patients suffering from IIH. The role that this feature plays in the etiology of IIH has been a matter of dispute. A lumped-parameter model of intracranial pressure dynamics that accommodates venous sinus stenosis via a Starling-like resistor has been developed to study the etiology, diagnosis, and treatment of IIH. In the absence of the Starling-like resistor, the system has a unique asymptotically-stable steady-state with normal pressures. With this type of resistor present, a second, asymptotically-stable steady-state may exist. This state is characterized by elevated ICP concurrent with a compressed venous sinus. It is hypothesized that IIH is a physiological manifestation of this elevated steady-state, that the primary cause of IIH is a compressible, as opposed to rigid, venous sinus, and that the observed stenosis is a necessary characteristic of the elevated steady-state. Simulations suggest a possible diagnostic technique for IIH, and the efficacy of various treatment options is examined. Results also indicate that the venous sinus stenosis may persist even after treatment has reduced the elevated ICP of IIH.
\end{abstract}

\section{Introduction}

Idiopathic intracranial hypertension (IIH), also called pseudotumor cerebri and benign intracranial hypertension, is a syndrome of unknown cause characterized by elevated intracranial pressure (ICP) without evidence of ventricular dilatation, mass lesion, or dural sinus thrombosis. It presents with symptoms of headache, 
nausea, vomiting, papilledema, and visual obscurations [1]. In most patients suffering from IIH, a stenosis or tapering of the lateral sinuses is observed by magnetic resonance venography or retrograde catheter venography [2-6]. Prior to the present modeling effort, the role played by venous sinus stenosis in the etiology of IIH has been unresolved and a matter of some dispute.

A lumped-parameter model of intracranial pressure dynamics is utilized to explore the relationship between IIH and lateral sinus stenosis. In previous models of this type [7-10] the sinuses have been considered rigid so as to withstand the negative transmural pressure normally endured by these vessels. In the present work, the model in [9] has been modified so that this assumption is no longer made. Specifically, the resistance to venous sinus drainage is formulated so as to be sensitive to transmural pressure changes. This modification now allows for partial collapse of the lateral sinuses in the face of elevated ICP with a resulting increase in resistance to venous sinus drainage.

\section{The lumped-parameter model}

A diagram of the lumped-parameter model that has been enhanced for this study is shown in figure 1. Previously, this model has been used to study steady-state intracranial pressures in microgravity [9]. A brief description of the model is given below. A full description that includes calibration of the normal parameter values is given in [9].

\subsection{Features of the mathematical model}

Cerebral blood flow $\left(Q_{I C}\right)$ and CSF formation by the choroid plexus $\left(Q_{C F}\right)$ are considered constant in the current application. Filtration from the intracranial capillaries across the blood-brain barrier $\left(Q_{C B}\right)$ is modeled by the Starling-Landis equation involving both hydrostatic and osmotic forces. All other flows are related to pressure differences by

$$
Q_{i j}=\left(P_{i}-P_{j}\right) / R_{i j}=Z_{i j}\left(P_{i}-P_{j}\right),
$$

where $Q_{i j}$ is the flow from compartment $i$ to compartment $j, P_{i}$ and $P_{j}$ are the spatially-averaged pressures of compartments $i$ and $j$ respectively, $R_{i j}$ is the lumped resistance, and $Z_{i j}$ is the fluidity (inverse of $R_{i j}$ ). Volume adjustments between adjacent compartments are related to changes in pressures by local compliance parameters $C_{i j}=C_{j i}$. Applying the law of conservation of mass in compartments I,C,S,F,B, and T results in a set of governing differential equations defined in matrix form by

$$
C \frac{d P}{d t}+Z P=Q
$$

where $P=\left[P_{I}, P_{C}, P_{S}, P_{F}, P_{B}, P_{T}\right]^{t r}, C$ is a compliance matrix, $Z$ is a fluidity matrix, and $Q$ is a vector of forcing terms that involves extra-cranial pressures and known flows. The matrix equation (2) may appear to be linear, but entries in the 


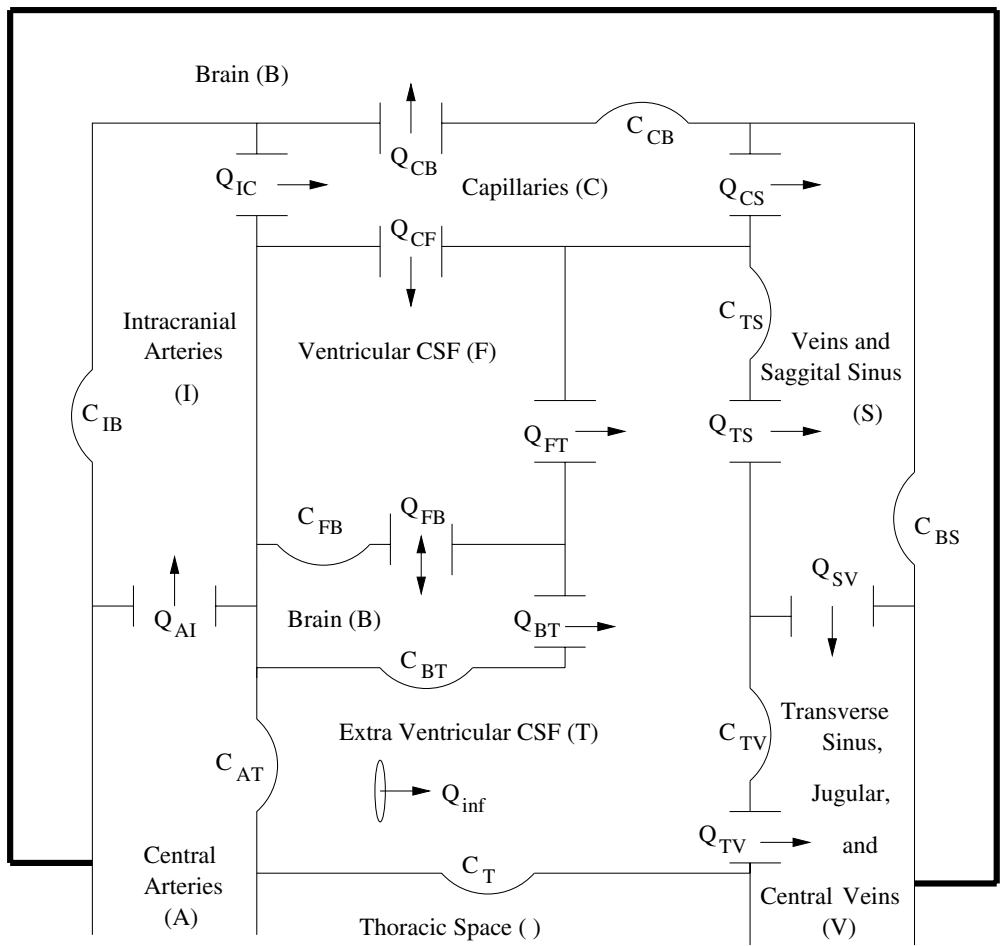

Figure 1: A diagram of the lumped-parameter model. The dark line represents the rigid cranial wall. $Q_{i j}$ represents fluid flow from compartment $i$ to compartment $j$. Arrows indicate the customary direction of flow. $Q_{i n f}$ represents an infusion rate of CSF. $C_{i j}$ represents a distensible surface between compartments $i$ and $j$. The Starling-like resistor described in this paper is associated with $Q_{S V}$.

compliance and fluidity matrices will be functions of compartmental pressures and time, introducing nonlinearities.

If the oscillatory effects of the forcing terms in $Q$ are subtracted, the solution of (2) is a set of time-dependent intracranial pressures averaged over each cardiac cycle. It was shown [9] that if the fluidity terms in $Z$ are all constants then all solutions of (2) will tend to a unique steady state. For the current investigation,the fluidity term $Z_{S V}$ is no longer constant but is allowed to be pressure sensitive. With this modification, convergence to a unique steady-steady state is no longer guaranteed, and extensive analysis of the various possible steady-states and their stability properties is required. 


\subsection{The Starling-like resistor}

A Starling resistor in a model for fluid flow through a collapsible vessel is a resistance term that is dependant upon transmural pressure. When transmural pressure is large, the vessel is considered open and the resistance to flow is small. Conversely, if the transmural pressure is small or negative, then the vessel is partially or completely collapsed resulting in a large (or possibly infinite) resistance. In many models [10-12], a Starling resistor has been placed at the location where the cerebral veins empty into the saggital sinus near the cerebral lacunae. A common feature of these locations is that the transmural pressure is positive in the normal resting state. Starling resistors have not previously been introduced into the venous sinuses because normal transmural pressure in these vessels is negative, and rigid walls were assumed to prevent collapse.

The observed stenosis of the lateral sinuses in IIH patients suggests that the venous sinuses are not completely rigid. Therefore, to allow for collapse the present model includes a Starling-like resistor dependent on transmural pressure at the level of the transverse sinus to represent the resistance to flow from compartment $\mathrm{S}$ to $\mathrm{V}$. The Starling-like resistor for the fluidity $Z_{S V}=1 / R_{S V}$ is defined in such a way that this fluidity term is positive, despite the transmural pressure $P_{V}-P_{T}$ being negative. However $Z_{S V}$ will decrease (resistance will increase) when $P_{V}-P_{T}$ becomes more negative. In particular, $Z_{S V}$ is defined by

$$
Z_{S V}=\operatorname{Max}\left[\bar{Z}_{S V}\left(1-m\left(P_{T V}-\bar{P}_{T V}\right)\right), p \bar{Z}_{S V}\right] \quad 0 \leq p \leq 1 .
$$

Here, $P_{T V}=P_{T}-P_{V}$, an overbar indicates the normal resting value, and Max refers to the larger of the two terms. The graph of the relationship between $Z_{S V}$ and $P_{T V}$ is piecewise-linear and continuous with an initial decreasing straight line segment of slope $-m \bar{Z}_{S V}$ that passes through the scale point $\left(\bar{P}_{T V}, \bar{Z}_{S V}\right)$, calibrated as in [9]. From equation (3), $p \bar{Z}_{S V}$ is the minimum value of $\bar{Z}_{S V}$, and beyond the pressure difference $P_{T V}$ that produces this minimum the graph of $Z_{S V}$ is a horizontal line at this minimum value. The form of this resistor is based on the results of [13] showing that when a compliant vessel with internal flow starts to collapse due to a uniformly applied external pressure it does so at the furthest downstream location, and when this occurs the degree of this collapse will be dependent upon the difference between the external and downstream pressures. It may well be the case that in healthy individuals no such Starling-like resistor is present and the sinuses are indeed rigid. This is achieved in the model by setting $m=0$, and the fluidity is therefore constant.

\section{Steady state analysis}

With the introduction of a pressure sensitive fluidity term $Z_{S V}$, a steady state, or equilibrium solution, to equation (2) for which $d P / d t=0$ is no longer constrained to be unique, and the number of steady state solutions may increase from unity, depending on the values of the parameters $m$ and $p$ in equation (3). The analysis in [14] shows that a piecewise linear curve $m=B(p)$ now divides the $p-m$ 
parameter plane into regions with unique and multiple steady states. This situation is shown in figure 2 . In the more rigid region of the $(p, m)$-plane, only one stable steady-state solution exists, namely the base-value steady state. This steady state solution is indicative of the normal healthy state. For values of $(p, m)$ in the less rigid region, three steady-states of the governing equations are found to exist; the original stable base-value state, an unstable saddle point, and a stable state of elevated pressures. The curve $m=B(p)$ separating the two domains of sinus rigidity is found in [14] to be a saddle-node bifurcation curve on which there are exactly two steady-states. Additionally, an upper bound on $m$, given by $m \leq M=B(1)$, must be imposed because if $m$ is allowed to exceed this value, then a trans-critical bifurcation would occur. For $m>M$, while the stable elevated pressure state in the less rigid region state remains stable, the stable base-value state becomes unstable and the unstable saddle-node state becomes stable. On physiological grounds, this possibility must be precluded.

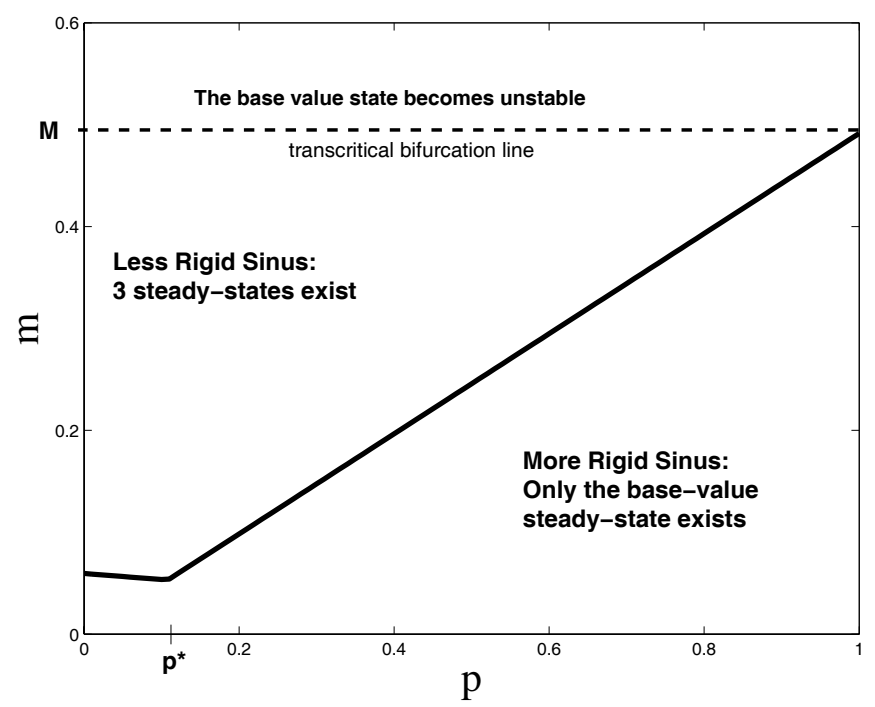

Figure 2: The $(p, m)$ parameter plane. The solid piecewise-linear curve represents the bifurcation curve $m=B(p)$. The dashed line is a transcritical bifurcation curve at the upper value $m=M$.

\section{Diagnosing IIH}

Simulations conducted in [15] show that if the transverse sinus is sufficiently collapsible, so that an elevated steady state exists in addition to the normal steady state, then a temporary precipitating event may be capable of causing a permanent transition from one stable steady state to the other. This result provides the basis 


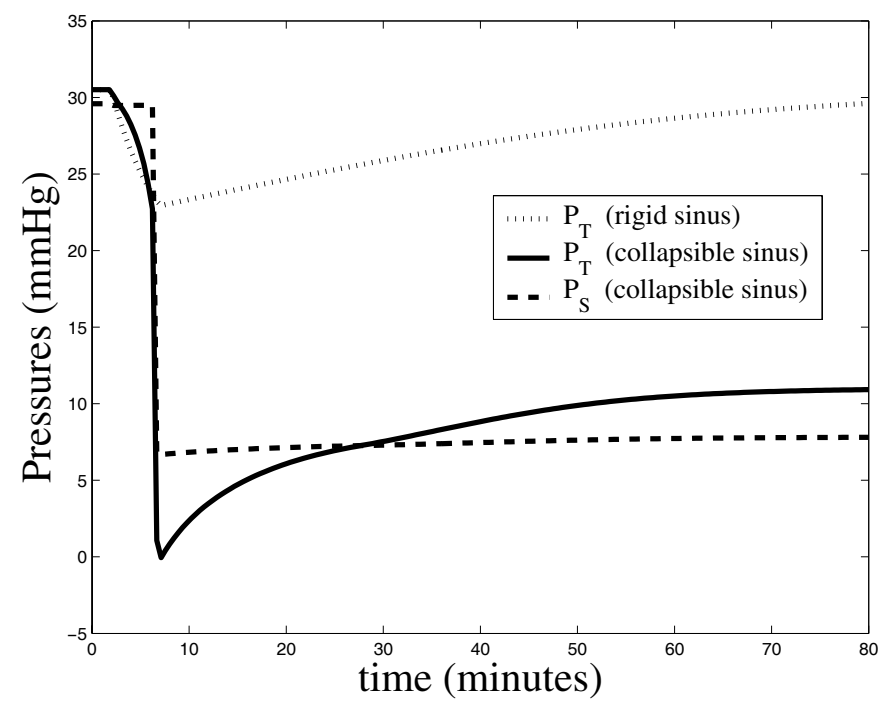

Figure 3: Simulated pressure responses to a CSF withdrawal of $5 \mathrm{ml} / \mathrm{min}$ for $5 \mathrm{~min}$ starting at $t=2 \mathrm{~min}$. Solid curve: CSF pressure $\left(P_{T}\right)$ with a collapsible sinus. Dashed curve: saggital sinus pressure $\left(P_{S}\right)$ with a collapsible sinus. For these simulations with a non-rigid sinus, $p=0.1, m=0.08$, and the initial conditions reflect the elevated steady-state. Dotted curve: CSF pressure with a rigid sinus. In this case the initial elevated condition is due to a CSF absorption blockage.

for a potential diagnostic technique that may determine if a patient either has IIH or is at risk for its development. For assessing the risk of developing IIH, the possibility of a transition from the normal pressure state to the elevated pressure state is relevant. For confirming a diagnosis of $\mathrm{IIH}$, the starting state will be the elevated steady state, and a transition to the normal state is desired. The proposed diagnostic technique involves monitoring the response of lumbar CSF pressure to a controlled change in CSF volume through withdrawal or infusion of CSF. As indicated in figure 1, this CSF volume change is introduced into the model through the flow $Q_{\text {inf }}$ in the extra-ventricular CSF compartment.

Figure 3 displays simulated responses to a $5 \mathrm{ml} / \mathrm{min} \mathrm{CSF}$ withdrawal over 5 minutes. If the sinus is collapsible and the initial conditions are those associated with the elevated state, the solid curve in figure 3 predicts that this CSF withdrawal initiates a long term transition to the normal state. The rapid pressure drops evident in figure 3 have been observed in clinical experience with IIH patients after CSF removal. For comparison purposes, simulations were also run to predict the effect of this same CSF withdrawal on an elevated pressure state due to a legitimate CSF drainage blockage when the sinus is rigid $(m=0)$. In this latter case, as shown 


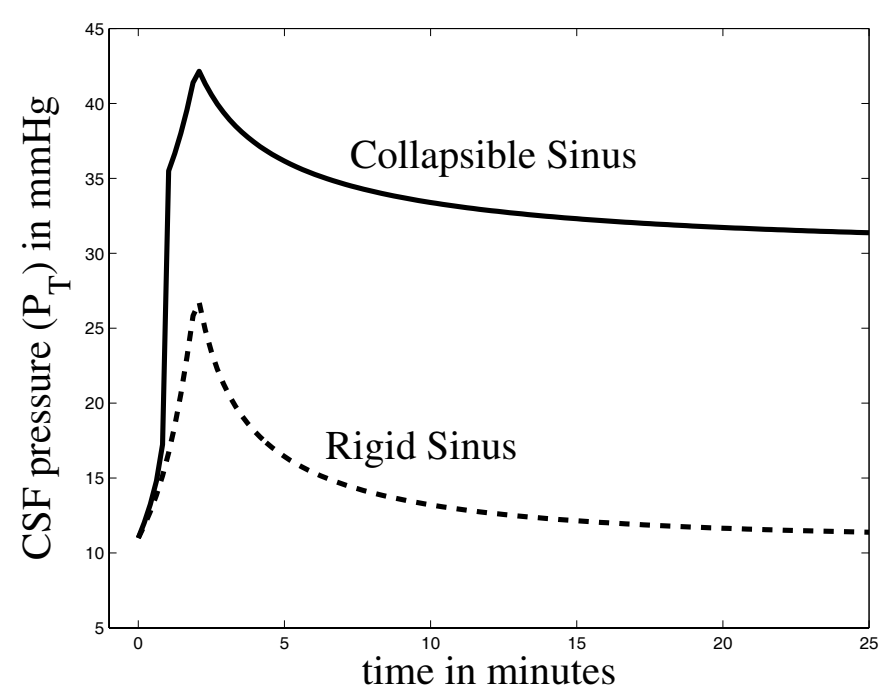

Figure 4: Response of the CSF pressure $P_{T}$ to a $14 \mathrm{ml}$ bolus infusion over $2 \mathrm{~min}$ utes. Solid curve: $m=0.08$ and $p=0.1$, representing a collapsible sinus. Dotted curve: $m=0$, representing a fully rigid sinus.

by the dotted line in figure 3 , the CSF withdrawal causes only a temporary drop in CSF pressure followed by a slow return to the hypertensive state.

Figure 4 displays the simulated responses to a $7 \mathrm{ml} / \mathrm{min}$ CSF infusion over 2 minutes. As shown by the solid curve in figure 4 , this temporary infusion is sufficient to cause a transition to the elevated state when there is a collapsible sinus. These simulation results are in full accord with the clinical findings of Higgins and Pickard [5] who reported that after an IIH patient was successfully treated with a shunt implant, the patient's CSF pressure still exhibited a "rapid" increase from normal in response to infusion. However, as indicated by the dotted curve in figure 4 , when the sinus is fully rigid $(m=0)$, the pressure response is the expected quick increase in $P_{T}$ followed by a gradual decay to the normal steady-state [16].

These time-dependent simulations indicate that a rapid and sustained transition from a normal to an elevated steady state in response to a CSF infusion is a key predictor of the presence of a compressible transverse sinus and susceptibility to IIH. Similarly, for a patient with elevated ICP, a rapid and sustained transition to the normal pressure state following a CSF withdrawal indicates the presence of a compressible transverse sinus and confirms a diagnosis ofIIH. If the elevated ICP is due to some other cause, a return to the hypertensive state following CSF withdrawal is to be expected. 


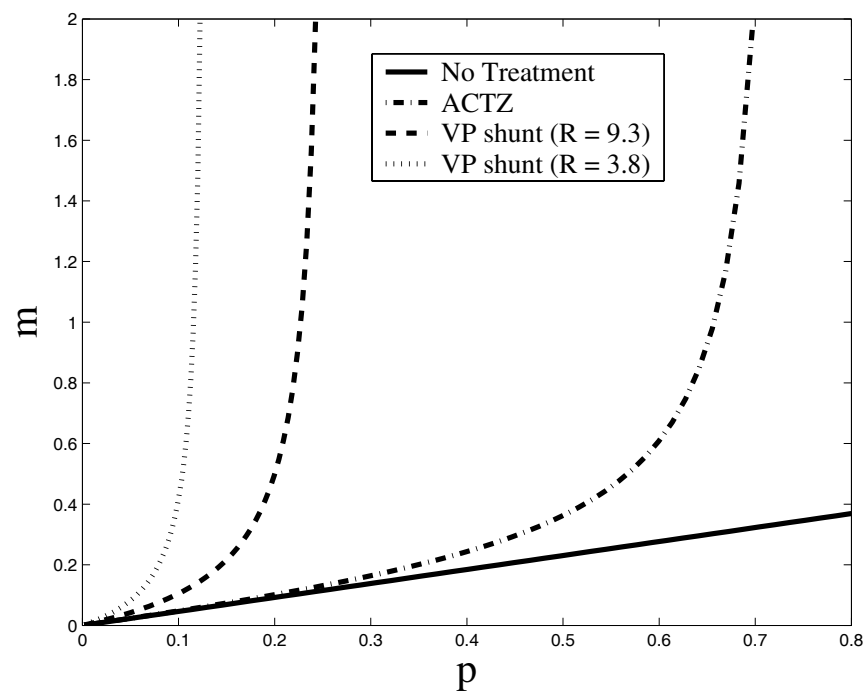

Figure 5: The altered $(p, m)$-plane with treatment applied. A stable, elevated steady-state exists only for parameters falling above (or to the left of) the given curves. Solid line: no simulated treatment, as in figure 2. Dash-Dot curve: CSF production cut in half (ACTZ). Dashed curve: large resistance $(\mathrm{R}=9.3 \mathrm{mmHg} /(\mathrm{ml} / \mathrm{min})) \mathrm{VP}$ shunt. Dotted curve: small resistance $(\mathrm{R}=3.8 \mathrm{mmHg} /(\mathrm{ml} / \mathrm{min})) \mathrm{VP}$ shunt.

\section{Simulating the effects of two treatment methods}

Figure 2 shows that an elevated steady state exists only for an ordered pair of parameters in the Starling-like resistor that fall above and to the left of the straight line that is a bifurcation curve in the $(p, m)$-plane. Simulations incorporating modifications that model the effects of ACTZ (a reduction in CSF production) and a ventriculoperitoneal (VP) shunt (CSF diversion) predict that these treatment modalities will significantly alter the location and shape of the dividing bifurcation curve. The altered bifurcation curves in the $(p, m)$-plane predicted by the simulations are shown in figure 5. It can be seen that ACTZ significantly reduces the region of the $(p, m)$-plane in which an elevated steady state can exist. Shunting reduces this multiple steady state region to an even greater extent, with the lower resistance shunt almost entirely eliminating the region where a stable elevated steady state can exist. A second indication of treatment effectiveness is given by figure 6 . It can be seen from this figure that if ACTZ does not entirely eliminate the elevated steady state by shifting the bifurcation curve in the $(p, m)$-plane, then it does little to reduce the level of hypertension. On the other hand, both types of VP shunts reduce the level of hypertension significantly, with the lower resistance shunt producing the best results. In addition to the VP shunts, idealized lumboperi- 


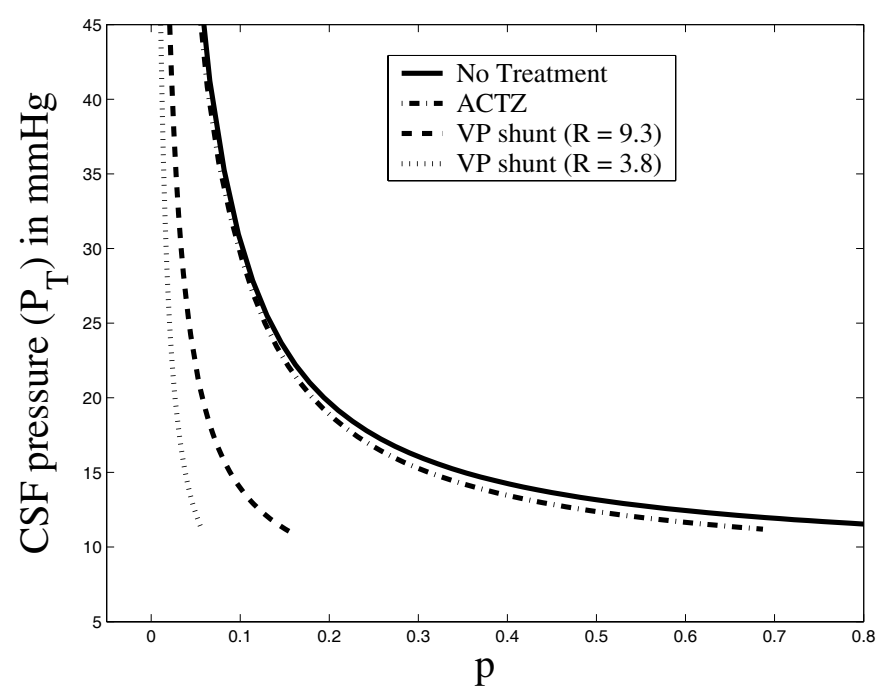

Figure 6: Elevated steady state values of $P_{T}$ in terms of $p$. Solid curve: no simulated treatment, as in figure 2. Dash-Dot curve: CSF production cut in half (ACTZ). Dashed curve: large resistance $(\mathrm{R}=9.3 \mathrm{mmHg} /(\mathrm{ml} / \mathrm{min}))$ VP shunt. Dotted curve: small resistance $(\mathrm{R}=3.8 \mathrm{mmHg} /(\mathrm{ml} / \mathrm{min})) \mathrm{VP}$ shunt.

toneal (LP) shunts were also simulated using the extended model. The associated curves predicted for the LP shunts were found to be nearly indistinguishable from those for the VP shunts in figures 5 and 6 and will not be separately presented here.

\section{Resolving the venous sinus stenosis}

A number of studies $[2,5,6,17]$ have reported a disapearance of the venous sinus stenosis in IIH patients following CSF drainage or diversion procedures that cause a transition to the normal pressure state. More recently, however, Bono et al. [18] report that transverse sinus stenosis in their study persists even after CSF pressure has been reduced in all patients that presented with a stenosis. Results obtained by Stevens et al. [14] analyzing the present model as a dynamical system indicate that these findings are not contradictory. It is proved in [14] that the elevated pressure state and the maximally-stenosed sinus must occur simultaneously, regardless of the magnitude of the pressure elevation. Consequently, if the elevated ICP of IIH is reduced to near-normal levels by weight loss or some other intervention but the patient still remains in an elevated rather than normal pressure state, the sinus will remain in its maximally collapsed state. Even though clinically it may appear that pressures are now normal, if they are merely reduced but there has not been an actual transition to the normal pressure state, the associated stenosis will not resolve. 


\section{Concluding remarks}

A steady-state analysis of the current model demonstrates that when the transverse sinus is not required to be rigid an additional stable steady-state with elevated pressures may be present as a solution of the system. It is hypothesized that IIH is a physiological manifestation of this additional stable elevated pressure state, and that a requirement for the development of IIH is a collapsible, as opposed to fully rigid, transverse sinus. Simulations suggest that rapid transitions between normal and elevated states in response to CSF infusions or withdrawals are indicative of the presence of such collapsible vessels. The model also makes predictions concerning the relative efficacy of standard treatment methods for IIH in various parameter regimes. As with any modeling study, measured data from IIH patients will be required to fully validate the present hypotheses.

\section{References}

[1] Binder, D., Horton, J., Lawton, M. \& McDermott, M., Idiopathic intracranial hypertension. Neurosurgery, 54(3), pp. 538-540, 2004.

[2] Baryshnik, D. \& Farb, R., Changes in the appearance of the venous sinuses after treatment of disordered intracranial pressure. Neurology, 62, pp. 144546, 2004.

[3] Farb, R., Vanek, I., Scott, J., Mikulis, D., Willinsky, R., Tomlinson, G. \& terBrugge, K., Idiopathic intracranial hypertension: The prevalence and morphology of sinovenous stenosis. Neurology, 60, pp. 1418-1424, 2003.

[4] Higgins, J., Gillard, J., Owler, B., Harkness, K. \& Pickard, J., Mr venography in idiopathic intracranial hypertension: unappreciated and misunderstood. $J$ Neruol Neurosurg Psychiatry, 75, pp. 621-25, 2004.

[5] Higgins, J. \& Pickard, J., Lateral sinus stenosis in idiopathic intracranial hypertension resolving after csf diversion. Neurology, 62, pp. 1907-8, 2004.

[6] King, J., Mitchell, P., Thomson, K. \& Tress, B., Manometry combined with cervical puncture in idiopathic intracranial hypertension. Neurology, 58, pp. 26-30, 2002.

[7] Sorek, S., Bear, J. \& Karni, Z., A non-steady compartmental flow model of the cerebrovascular system. J Biomechanics, 21, pp. 695-704, 1988.

[8] Stevens, S. \& Lakin, W., Local compliance effects on the global csf pressurevolume relationship in models of intracranial pressure dynamics. Mathematical and Computer Modelling of Dynamical Systems, 6(4), pp. 445-465, 2001.

[9] Stevens, S., Lakin, W. \& Penar, P., Modeling steady-state intracranial pressures in supine, head-down tilt, and microgravity conditions. Aviat Space Environ Med, 76, pp. 329-338, 2005.

[10] Ursino, M., A mathematical study of human intracranial hydrodynamics. part 1 - the cerebrospinal fluid pulse pressure. Annals of Biomedical Engineering, 16, pp. 379-401, 1988.

[11] Czosnyka, M., Piechnik, S., Richards, S., Kirkpatrick, P., Smielewski, P. \& Pickard, J., Contribution of mathematical modelling to the interpretation of 
bedside tests of cerebrovascular autoregulation. J Neurol Neurosurg Psychiatry, 63, pp. 721-731, 1997.

[12] Piechnik, S., Czosnyka, M., Richards, H., Whitfield, P. \& Pickard, J., Cerebral venous blood outflow: A theoretical model based on laboratory simulation. Neurosurgery, 49, pp. 1214-23, 2001.

[13] Heil, M., Stokes flow in collapsible tubes: computation and experiment. $J$ Fluid Mech, 353, pp. 285-312, 1997.

[14] Stevens, S., Previte, M., Lakin, W., Thakore, N., Penar, P. \& Hamschin, B., Idiopathic intracranial hypertension and transverse sinus stenosis: a modeling study. Math Med Biol, 24, pp. 85-109, 2007.

[15] Stevens, S., Thakore, N., Lakin, W., Penar, P. \& Tranmer, B., A modeling study of idiopathic intracranial hypertension: etiology and diagnosis. Neurological Res, 2007 (in press).

[16] Marmarou, A., Shulman, K. \& Rosende, R., A nonlinear analysis of the cerebrospinal fluid system and intracranial pressure dynamics. J Neurosurg, $\mathbf{4 8}$, pp. 332-344, 1978.

[17] McGonigal, A., Bone, I. \& Teasdale, E., Resolution of transverse sinus stenosis in idiopathic intracranial hypertension after l-p shunt. Neurology, 62(3), pp. 514-5, 2004.

[18] Bono, F., Gilberto, C., Mastrandrea, C., Cristiano, D., Lavano, A., Fera, F. \& Quattrone, A., Transverse sinus stenoses persist after normalization of the csf pressure in ideopathic intracranial hypertension. Neurology, 65, pp. 10901093, 2005. 